07

\title{
Модуль объемного сжатия крупно- и нанокристаллического сульфида серебра
}

\author{
() С.И. Садовников
}

Институт химии твердого тела Ур РАН, Екатеринбург, Россия

E-mail: sadovnikov@ihim.uran.ru

\section{(Поступила в Редакцию 17 апреля 2018 г.)}

С использованием экспериментальных данных по температурным зависимостям теплоемкости и коэффициента термического расширения крупно- и нанокристаллического сульфидов серебра определено изменение модуля объемного сжатия $B$ в области температур $300-960 \mathrm{~K}$. Установлено, что в изученной области температур модуль объемного сжатия нанокристаллического сульфида серебра по величине меньше, чем модуль $B$ крупнокристаллического сульфида.

DOI: 10.21883/FTT.2018.12.46730.110

\section{1. Введение}

В последнее время в связи с возможностью использования в микро- и наноэлектронике активно изучается нанокристаллический сульфид серебра $[1,2]$. Сульфид серебра является полупроводником при температуре ниже $450 \mathrm{~K}$ и суперионным проводником при температуре больше $452 \mathrm{~K}$, и имеет три полиморфные модификации: полупроводниковый моноклинный акантит $\alpha-\mathrm{Ag}_{2} \mathrm{~S}$ при $T \lesssim 451 \mathrm{~K}$, суперионный объемно-центрированный (оцк) аргентит $\beta-\mathrm{Ag}_{2} \mathrm{~S}$ при температуре $452-859 \mathrm{~K}$ и суперионный гранецентрированный (гцк) $\gamma-\mathrm{Ag}_{2} \mathrm{~S}$ при температуре от $\sim 860 \mathrm{~K}$ до температуры плавления [3]. Применение нанокристаллического сульфида серебра перспективно для создания энергонезависимых устройств памяти и резистивных переключателей на основе гетероструктур $\mathrm{Ag}_{2} \mathrm{~S} / \mathrm{Ag}$, действие которых основано на обратимом превращении полупроводникового акантита $\alpha-\mathrm{Ag}_{2} \mathrm{~S}$ в суперионный аргентит $\beta-\mathrm{Ag}_{2} \mathrm{~S}$ с образованием проводящего канала из серебра $\mathrm{Ag}$ и аргентита $\beta-\mathrm{Ag}_{2} \mathrm{~S}[4-6]$.

Для применения нанокристаллического сульфида серебра в электронике нужны сведения о его теплофизических и упругих свойствах в интервале температур, охватывающем по меньшей мере области существования акантита и аргентита. Недавно термическое расширение крупно- и нанокристаллического сульфида серебра в области температур 300-980 К было изучено в серии работ [7-11]. Сведения об упругих свойствах крупнокристаллического сульфида серебра крайне ограничены. Согласно [12], сжимаемость $\kappa=1 / B$ ( $B$ - модуль объемного сжатия) крупнокристаллического акантита $\alpha-\mathrm{Ag}_{2} \mathrm{~S}$ при $273 \mathrm{~K}$ равна $2.9 \cdot 10^{-11} \mathrm{~m}^{2} \cdot \mathrm{N}^{-1}$ $(B=34.5 \mathrm{GPa})$. По данным сайта [13] модуль объемного сжатия $B$ крупнокристаллического акантита составляет от 26 до $34 \mathrm{GPa}$. Экспериментальные данные об упругих свойствах нанокристаллического сульфида серебра и их температурных зависимостях в литературе отсутствуют.
В связи с этим в настоящей работе с использованием ранее полученных данных [10,11] по температурным зависимостям теплоемкости $C_{p}(T)$ и коэффициента термического расширения $\alpha(T)$ крупно- и нанокристаллического сульфидов серебра составов $\mathrm{Ag}_{2} \mathrm{~S}$ и $\mathrm{Ag}_{1.93} \mathrm{~S}$ [14-16] соответственно будут найдены температурные зависимости модуля объемного сжатия трех фаз сульфида серебра в крупно- и нанокристаллическом состояниях.

\section{2. Оценка модуля объемного сжатия поликристаллического сульфида серебра}

Линейный коэффициент термического расширения $\alpha(T)$ связан с молярной теплоемкостью $C_{V}(T)$ известным соотношением [17]

$$
\alpha(T)=\frac{\gamma}{3 B} \frac{C_{V}(T)}{v_{m}},
$$

где $B$ - модуль объемного сжатия (bulk modulus), $v_{m}=M / \rho-$ молярный объем, $M-$ молярная масса, $\rho$ - плотность,

$$
\gamma=\left[\sum_{\mathbf{k}} \gamma_{\mathbf{k}} C_{V}(\mathbf{k})\right] /\left[\sum_{\mathbf{k}} C_{V}(\mathbf{k})\right]
$$

- постоянная Грюнайзена (полный параметр Грюнайзена),

$$
\gamma_{\mathbf{k}}=-\left(V / \omega_{\mathbf{k}}\right)\left[\partial\left(\omega_{\mathbf{k}}\right) / \partial V\right]=-\partial\left(\ln \omega_{\mathbf{k}}\right) \partial(\ln V)
$$

- параметр Грюнайзена для колебательной моды с волновым вектором $\mathbf{k}$ и угловой частотой $\omega_{\mathbf{k}}=c k, c-$ скорость распространения упругих колебаний в решетке, $k=|\mathbf{k}|$. В случае поликристалла постоянная Грюнайзена является величиной, усредненной по частотам колебаний решетки кристалла. При наличии данных об $\alpha(T)$, 
Рентгеновская плотность $\rho$ и молярный объем $v_{m}$ фаз $\alpha, \beta$ и $\gamma$ сульфида серебра в крупно- и нанокристаллическом состояниях

\begin{tabular}{c|c|c|c|c|c|c}
\hline \multirow{2}{*}{ Фаза } & \multicolumn{3}{|c|}{ Крупнокристаллическая } & \multicolumn{3}{c}{ Нанокристаллическая } \\
\cline { 2 - 7 } & $\alpha-\mathrm{Ag}_{2} \mathrm{~S}$ & $\beta-\mathrm{Ag}_{2} \mathrm{~S}$ & $\gamma-\mathrm{Ag}_{2} \mathrm{~S}$ & $\alpha-\mathrm{Ag}_{1.93} \mathrm{~S}$ & $\beta-\mathrm{Ag}_{1.93} \mathrm{~S}$ & $\gamma-\mathrm{Ag}_{1.93} \mathrm{~S}$ \\
\hline$\rho, \mathrm{g} \cdot \mathrm{cm}^{-3}$ & 7.25 & 7.22 & 7.20 & 6.97 & 6.95 & 6.94 \\
$v_{m} \cdot 10^{6}, \mathrm{~m}^{3} \cdot \mathrm{mol}$ & 34.18 & 34.33 & 34.42 & 34.47 & 34.57 & 34.62
\end{tabular}

$C_{V}(T), \gamma$ и $v_{m}$ из соотношения (1) можно определить модуль объемного сжатия как

$$
B(T)=\frac{\gamma}{3 v_{m}} \frac{C_{V}(T)}{\alpha(T)}
$$

В адиабатической калориметрии твердых веществ экспериментально измеряемой величиной является $C_{p}$, поэтому при использовании измеренной теплоемкости нужно учитывать поправку $\left(C_{p}-C_{V}\right)$, составляющую около $1 \%$ от величины $C_{p}$ :

$$
C_{p}-C_{V}=9 \alpha^{2} v_{m} B T .
$$

С учетом поправки (3) модуль объемного сжатия имеет вид

$$
B(T)=\frac{\gamma}{3 v_{m}} \frac{C_{p}(T)}{\alpha(T)[1+3 \gamma \alpha(T)]} .
$$

Как отмечено, в температурном интервале от 300 до температуры плавления $\sim 1100 \mathrm{~K}$ сульфид серебра имеет три полиморфные модификации: низкотемпературную моноклинную фазу $\alpha-\mathrm{Ag}_{2} \mathrm{~S}$ (акантит), объемноцентрированную (оцк) фазу $\beta-\mathrm{Ag}_{2} \mathrm{~S}$ (аргентит) и высокотемпературную гранецентрированную (гцк) фазу $\gamma-\mathrm{Ag}_{2} \mathrm{~S}$.

Плотность фаз при $300 \mathrm{~K}$, необходимую для оценки величины молярного объема $v_{m}$, определяли как

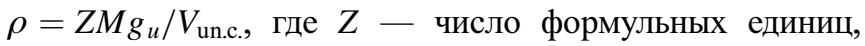
приходящихся на элементарную ячейку $(Z=4$ для фаз $\alpha-\mathrm{Ag}_{2} \mathrm{~S}$ и $\gamma-\mathrm{Ag}_{2} \mathrm{~S}$, и $Z=2$ для фазы $\left.\beta-\mathrm{Ag}_{2} \mathrm{~S}\right), M-$ молекулярная масса фазы, $g_{u}=1.66 \cdot 10^{-24} \mathrm{~g}$ - единица атомной массы, $V_{\text {un.c. }}$ - объем элементарной ячейки при $300 \mathrm{~K}$. Параметры моноклинных элементарных ячеек крупнокристаллического $\alpha-\mathrm{Ag}_{2} \mathrm{~S}$ и нанокристаллического $\alpha-\mathrm{Ag}_{1.93} \mathrm{~S}$ акантита при $300 \mathrm{~K}$, необходимые для определения объема элементарной ячейки, приведены в работах [14,15] соответственно.

Для определения молярного объема оцк сульфида серебра - аргентита $\beta-\mathrm{Ag}_{2} \mathrm{~S}$, - нужно экстраполировать температурную зависимость периода решетки на $300 \mathrm{~K}$. Температурная зависимость периода решетки $a_{\text {arg }}(T)$ крупнокристаллического аргентита $\beta-\mathrm{Ag}_{2} \mathrm{~S}$, построенная по результатам работ $[7,8]$ и проведенных дополнительных высокотемпературных дифракционных измерений при температуре до $773 \mathrm{~K}$, является нелинейной (рис. 1) и в интервале $443-673 \mathrm{~K}$ описывается полиномом

$$
a_{\mathrm{arg}}(T)=a_{0}+a_{1} T+a_{2} T^{2},
$$

где $a_{0}=0.48592 \mathrm{~nm}, \quad a_{1}=-1.0803 \cdot 10^{-5} \mathrm{~nm} \cdot \mathrm{K}^{-1}$ и $a_{2}=2.4879 \cdot 10^{-8} \mathrm{~nm} \cdot \mathrm{K}^{-2}$. В соответствии с этим пе- риод решетки $a_{\arg }(300)$ крупнокристаллического аргентита $\beta-\mathrm{Ag}_{2} \mathrm{~S}$, экстраполированный на $300 \mathrm{~K}$, равен $0.48492 \mathrm{~nm}$. Для нанокристаллического аргентита $\beta-\mathrm{Ag}_{1.93} \mathrm{~S}$ период решетки $a_{\mathrm{arg}}(300) \approx 0.486 \mathrm{~nm}$.

Период а элементарной ячейки гцк фазы $\gamma-\mathrm{Ag}_{2} \mathrm{~S}$ при $923 \mathrm{~K}$ равен $0.6283 \mathrm{~nm} \mathrm{[18].} \mathrm{Период} \mathrm{a} \mathrm{фазы} \gamma-\mathrm{Ag}_{2} \mathrm{~S}$, экстраполированный на $300 \mathrm{~K}$, равен $0.6114 \mathrm{~nm}$. Для нанокристаллического сульфида серебра $\gamma-\mathrm{Ag}_{1.93} \mathrm{~S}$ период $a$, экстраполированный на $300 \mathrm{~K}$, равен $0.6125 \mathrm{~nm}$.

Плотности и молярные объемы трех фаз сульфида серебра в крупно- и нанокристаллическом состояниях, рассчитанные с учетом представленных параметров элементарных ячеек, приведены в таблице.

В литературе нет данных о величине постоянной Грюнайзена $\gamma$ какой-либо фазы сульфида серебра $\mathrm{Ag}_{2} \mathrm{~S}$. Сведения о скоростях распространения упругих колебаний в решетке сульфида серебра, по которым можно оценить постоянную Грюнайзена $\gamma$, тоже отсутствуют. Косвенно о величине $\gamma$ сульфида серебра можно судить по данным об упругих свойствах двойного сульфида $\mathrm{AgGaS}_{2}$ [19-24], согласно которым постоянная Грюнайзена монокристалла $\mathrm{AgGaS}_{2}$ составляет от 0.43 до 1.0 при комнатной температуре и при повышении температуры до $700 \mathrm{~K}$ растет до 0.55-1.20. Постоянная Грюнайзена монокристалла сульфида галлия $\mathrm{GaS}$ при $300 \mathrm{~K}$ равна $\sim 0.7$ и слабо уменьшается с ростом температуры [25]. С учетом данных по $\mathrm{AgGaS}_{2}$ и $\mathrm{GaS}$ можно предположить, что постоянная Грюнайзена $\gamma$ сульфида

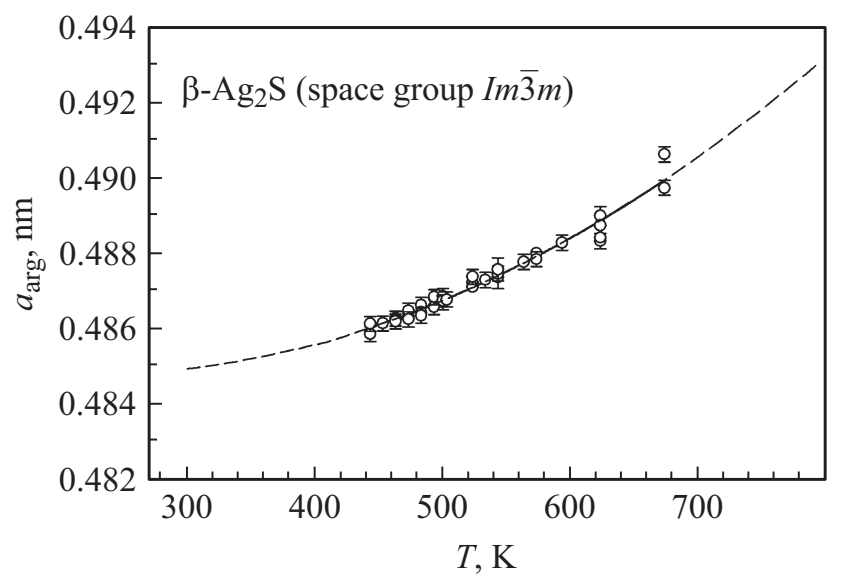

Рис. 1. Зависимость периода решетки $a_{\arg }$ аргентита $\beta-\mathrm{Ag}_{2} \mathrm{~S}$ от температуры $T$. Аппроксимация измеренного периода решетки $a_{\text {arg }}$ функцией (5) в область температур меньше 420 и больше $680 \mathrm{~K}$ показана штрихами. 
серебра при $300 \mathrm{~K}$ по величине близка к 1.0 и растет примерно на 5\% при увеличении температуры на $100 \mathrm{~K}$.

Согласно [26,27], постоянная Грюнайзена $\gamma$ связана с коэффицентом Пуассона (Poison ratio) $\mu$ следующим соотношением

$$
\gamma=3(1+\mu) /[2(2-3 \mu)]
$$

В работе [28] описан ab initio метод расчета упругих констант неорганических материалов. Результаты расчетов в виде базы данных по упругим свойствам неорганических соединений представлены в проекте [29]. В частности, для моноклинного (пр. гр. $P 2_{1} / c$ ) сульфида серебра $\mathrm{Ag}_{2} \mathrm{~S}$ коэффицент Пуассона $\mu$ составляет $\sim 0.13$ и более [30]. Для $\mu=0.13$ из соотношения (6) следует, что постоянная Грюнайзена $\gamma$ сульфида серебра равна $\sim 1.05$.

С учетом отмеченного для последующего расчета в первом приближении примем, что температурная зависимость постоянной Грюнайзена сульфида серебра имеет вид

$$
\gamma(T)=1.0+0.0005(T-300)
$$

Для определения температурных зависимостей модуля объемного сжатия $B(T)$ использовали данные по коэффициенту термического расширения $\alpha(T)$, измеренному в работах $[10,11]$ дилатометрическим методом, и по теплоемкости $C_{p}(T)$, измеренной в этих же работах на поликристаллических образцах крупно- и нанокристаллического сульфида серебра. Средний размер частиц в крупно- и нанокристаллическом сульфидах серебра составлял 430-460 и $\sim 60 \mathrm{~nm}$ соответственно [11].

Рассчитанные по уравнению (4) температурные зависимости модуля объемного сжатия $B(T)$ крупно- и нанокристаллического сульфидов серебра показаны на рис. 2 и 3.

С ростом температуры от 300 до $\sim 442 \mathrm{~K}$ модуль объемного сжатия $B$ крупнокристаллического акантита $\alpha-\mathrm{Ag}_{2} \mathrm{~S}$ снижается от $\sim 49.5$ до $\sim 47.6 \mathrm{GPa}$. При переходе от акантита $\alpha-\mathrm{Ag}_{2} \mathrm{~S}$ к аргентиту $\beta-\mathrm{Ag}_{2} \mathrm{~S}$ величина $B$ резко падает до $\sim 30 \mathrm{GPa}$ и при последующем росте температуры от $\sim 490$ до $\sim 856 \mathrm{~K}$ уменьшается до $\sim 22 \mathrm{GPa}$. Переход от аргентита $\beta-\mathrm{Ag}_{2} \mathrm{~S}$ к фазе $\gamma-\mathrm{Ag}_{2} \mathrm{~S}$ сопровождается небольшим снижением $B$ до $\sim 19.8 \mathrm{GPa}$ и последующим слабым уменьшением модуля объемного сжатия при увеличении температуры от $900 \mathrm{~K}$ (рис. 2). Оба перехода $\alpha-\mathrm{Ag}_{2} \mathrm{~S}-\beta-\mathrm{Ag}_{2} \mathrm{~S}$ и $\beta-\mathrm{Ag}_{2} \mathrm{~S}-\gamma-\mathrm{Ag}_{2} \mathrm{~S}$ происходят по механизму фазовых превращений первого рода $[10,11,31]$. Вместе с тем более резкое падение модуля объемного сжатия $B$ при переходе $\alpha-\mathrm{Ag}_{2} \mathrm{~S}-\beta-\mathrm{Ag}_{2} \mathrm{~S}$ по сравнению со следующим переходом $\alpha-\mathrm{Ag}_{2} \mathrm{~S}-\beta-\mathrm{Ag}_{2} \mathrm{~S}$ обусловлено некоторым различием этих переходов. Переход $\beta-\mathrm{Ag}_{2} \mathrm{~S}-\beta-\mathrm{Ag}_{2} \mathrm{~S}$ является превращением полупроводникового моноклинного сульфида в кубический суперионный сульфид. При переходе $\beta-\mathrm{Ag}_{2} \mathrm{~S}-\gamma-\mathrm{Ag}_{2} \mathrm{~S}$ происходит превращение оцк сульфида в гцк сульфид, причем обе фазы обладают суперионной проводимостью.

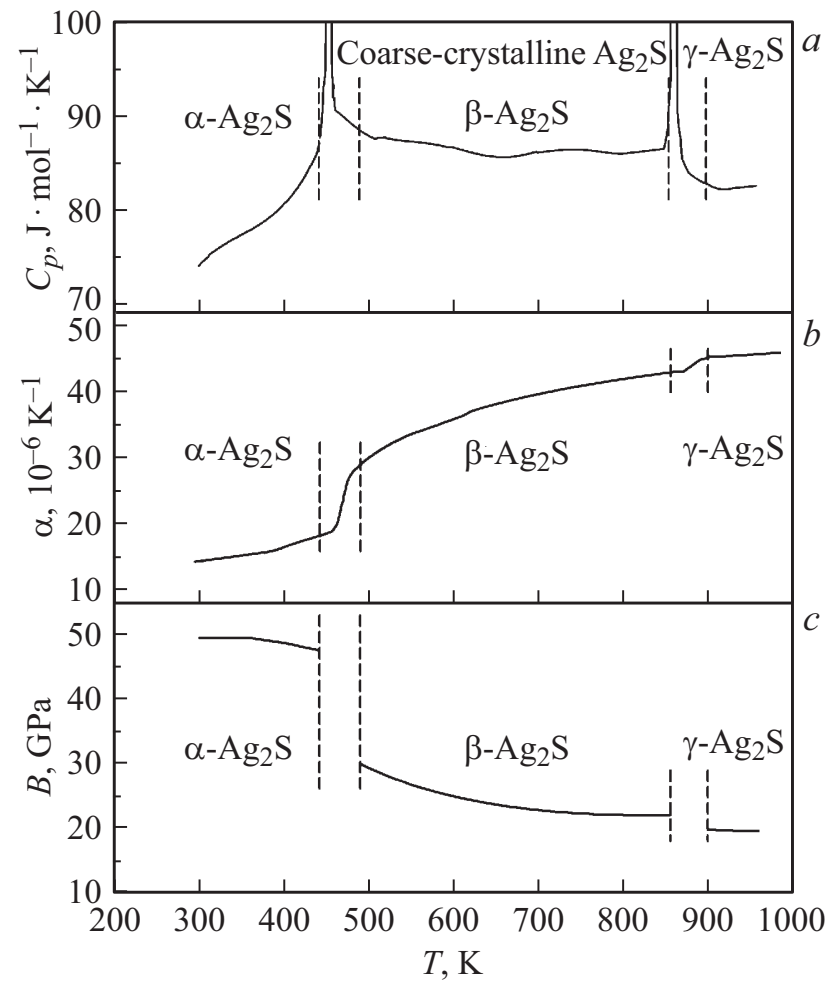

Рис. 2. Экспериментальные зависимости теплоемкости $C_{p}(T)(a)$ и коэффициента термического расширения $\alpha(T)(b)$ крупнокристаллического сульфида серебра $\mathrm{Ag}_{2} \mathrm{~S}$ [11] и рассчитанные зависимости модуля объемного сжатия $B$ от температуры $T(c)$ в областях существования акантита $\alpha-\mathrm{Ag}_{2} \mathrm{~S}$, аргентита $\beta-\mathrm{Ag}_{2} \mathrm{~S}$ и фазы $\gamma-\mathrm{Ag}_{2} \mathrm{~S}$. Области перехода между фазами $\alpha-\mathrm{Ag}_{2} \mathrm{~S}-\beta-\mathrm{Ag}_{2} \mathrm{~S}$ и $\beta-\mathrm{Ag}_{2} \mathrm{~S}-\gamma-\mathrm{Ag}_{2} \mathrm{~S}$ отмечены вертикальными штриховыми линиями.

Температурные зависимости модуля объемного сжатия крупнокристаллического сульфида серебра в областях существования моноклинной, оцк и гцк фаз описываются следующими функциями:

$$
\begin{gathered}
B_{\alpha-\mathrm{Ag}_{2} \mathrm{~S}}=33.65+98 \cdot 10^{-3} T-0.15 \cdot 10^{-3} T^{2} \\
(300<T<442 \mathrm{~K})[\mathrm{GPa}], \\
B_{\beta-\mathrm{Ag}_{2} \mathrm{~S}}(T)=73.57-0.13 T+0.08 \cdot 10^{-3} T^{2} \\
(490<T<856 \mathrm{~K}) \quad[\mathrm{GPa}], \\
B_{\gamma-\mathrm{Ag}_{2} \mathrm{~S}}(T)=22.73-3.30 \cdot 10^{-3} T \\
(900<T<960 \mathrm{~K}) \quad[\mathrm{GPa}] .
\end{gathered}
$$

Модуль объемного сжатия $B$ нанокристаллического акантита $\alpha-\mathrm{Ag}_{1.93} \mathrm{~S}$ при увеличении температуры от 300 до $\sim 442 \mathrm{~K}$ снижается от $\sim 35.6$ до $\sim 30.7 \mathrm{GPa}$. При переходе от акантита $\alpha-\mathrm{Ag}_{2} \mathrm{~S}$ к аргентиту $\beta-\mathrm{Ag}_{2} \mathrm{~S}$ величина $B$ скачком снижается до $\sim 26.9 \mathrm{GPa}$ и при последующем росте температуры от $\sim 470$ до $\sim 840 \mathrm{~K}$ уменьшается до $\sim 20.1 \mathrm{GPa}$. Переход $\beta-\mathrm{Ag}_{2} \mathrm{~S}-\gamma-\mathrm{Ag}_{2} \mathrm{~S}$ сопровождается небольшим снижением $B$ до $\sim 18.9 \mathrm{GPa}$ 
и последующим слабым уменьшением модуля объемного сжатия $B$ до $18.7 \mathrm{GPa}$ при увеличении температуры от 900 до $960 \mathrm{~K}$ (рис. 3).

Температурные зависимости $B(T)$ нанокристаллического сульфида серебра $\mathrm{Ag}_{1.93} \mathrm{~S}$ в областях существова-

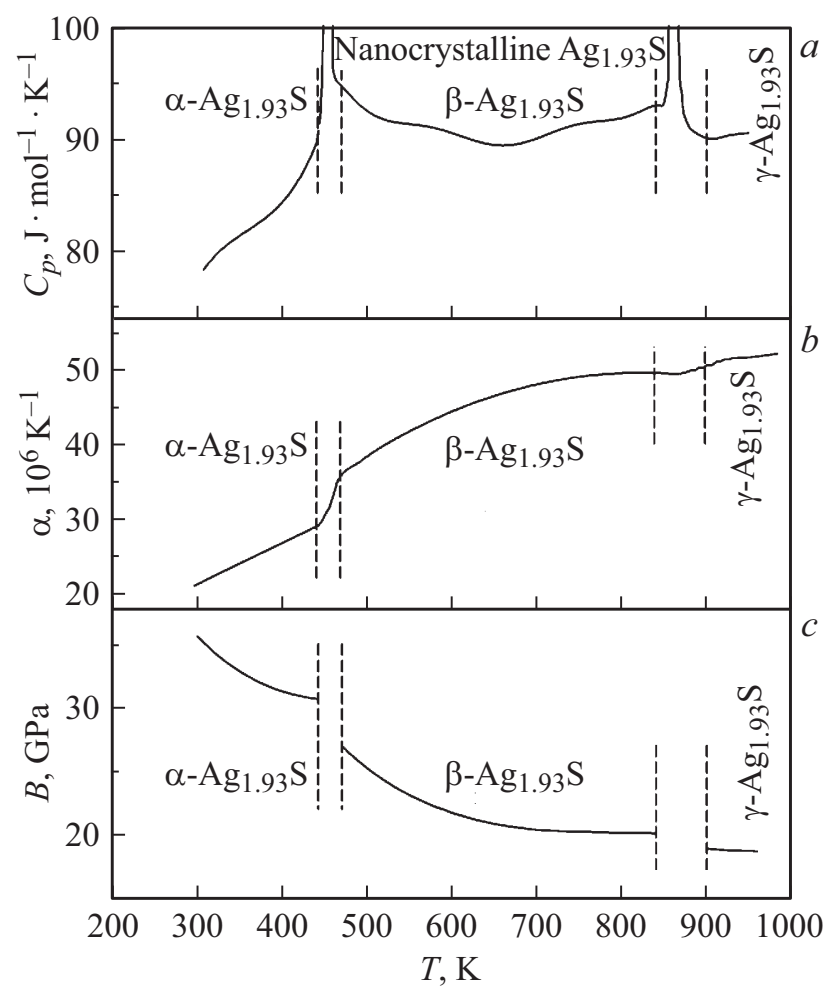

Pис. 3. Экспериментальные зависимости теплоемкости $C_{p}(T)(a)$ и коэффициента термического расширения $\alpha(T)(b)$ нанокристаллического сульфида серебра $\mathrm{Ag}_{1.93} \mathrm{~S}$ [11] и рассчитанные зависимости модуля объемного сжатия $B$ от температуры $T(c)$ в областях существования нанокристаллических фаз $\alpha-\mathrm{Ag}_{1.93} \mathrm{~S}, \beta-\mathrm{Ag}_{1.93} \mathrm{~S}$ и $\gamma-\mathrm{Ag}_{1.93} \mathrm{~S}$. Области перехода между фазами $\alpha-\mathrm{Ag}_{1.93} \mathrm{~S}-\beta-\mathrm{Ag}_{1.93} \mathrm{~S}$ и $\beta-\mathrm{Ag}_{1.93} \mathrm{~S}-\gamma-\mathrm{Ag}_{1.93} \mathrm{~S}$ отмечены вертикальными штриховыми линиями.

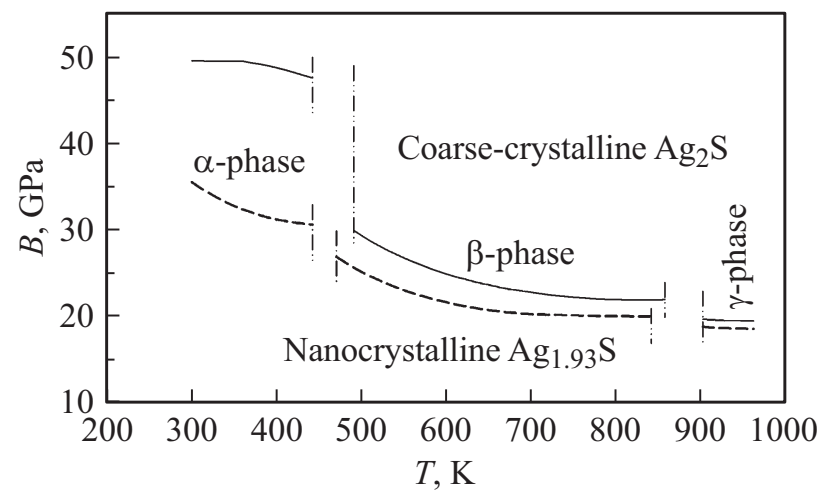

Рис. 4. Сравнение температурных зависимостей модуля объемного сжатия $B(T)$ крупно- и нанокристаллического сульфидов серебра в областях существования моноклинной $\alpha$-, оцк $\beta$ - и гцк $\gamma$-фаз. Области перехода между фазами $\alpha-\beta$ и $\beta-\gamma$ отмечены вертикальным штрихпунктиром. ния моноклинной, оцк и гцк фаз описываются следующими функциями:

$$
\begin{gathered}
B_{\alpha-\mathrm{Ag}_{1.93} \mathrm{~S}}(T)=74.46-194.6 \cdot 10^{-3} T+0.22 \cdot 10^{-3} T^{2} \\
(300<T<442 \mathrm{~K}) \quad[\mathrm{GPa}] \\
B_{\beta-\mathrm{Ag}_{1.93} \mathrm{~S}}(T)=65.34-0.12 T+0.08 \cdot 10^{-3} T^{2} \\
(470<T<840 \mathrm{~K}) \quad[\mathrm{GPa}] \\
B_{\gamma-\mathrm{Ag}_{1.93} \mathrm{~S}}(T)=21.74-3.21 \cdot 10^{-3} T \\
(900<T<960 \mathrm{~K}) \quad[\mathrm{GPa}] .
\end{gathered}
$$

Во всей области температур 300-960 К крупнокристаллический сульфид серебра имеет больший по величине модуль объемного сжатия $B$, чем нанокристаллический сульфида серебра (рис. 4). Наибольшая разница наблюдается в области существования моноклинной фазы со структурой типа акантита. Разница модуля $B$ в областях существования кубических фаз заметно меньше. Существенно меньшая величина $B$ нанокристаллического акантита обусловлена тем, что коэффициент термического расширения нанокристаллического акантита заметно больше и быстрее растет с ростом температуры, чем коэффициент термического расширения крупнокристаллического акантита (см. рис. 2,3).

\section{3. Заключение}

Определение температурных зависимостей модуля объемного сжатия $B$ крупно- и нанокристаллического сульфидов серебра в области температур 300-960 K показало, что величина модуля объемного сжатия $\alpha$-, $\beta$ и $\gamma$-фаз наносульфида серебра меньше, чем величины модуля $B$ этих же фаз крупнокристаллического $\mathrm{Ag}_{2} \mathrm{~S}$. Наибольшее влияние на величину $B(T)$ оказывает изменение коэффициента термического расширения. Для моноклинного акантита коэффициенты термического расширения и их температурные зависимости крупно- и нанокристаллического сульфидов серебра различаются в наибольшей степени, что приводит к существенно меньшей величине $B$ нанокристаллического акантита.

\section{Список литературы}

[1] A. Tang, Yu. Wang, H. Ye H, C. Zhou, C. Yang, X. Li, H. Peng, F. Zhang, Y. Hou, F. Teng. Nanotechnology 24, 355602 (2013).

[2] C. Cui, X. Li, J. Liu, Y. Hou, Y. Zhao, G. Zhong. Nanoscale Res. Lett. 10, 431 (2015).

[3] R.C. Sharma, Y.A. Chang. Bull. Alloy Phase Diagrams 7, 3, 263 (1986).

[4] K. Terabe, T. Hasegawa, T. Nakayama, M. Aono. Nature 433, 7021,47 (2005).

[5] S. Kaeriyama, T. Sakamoto, H. Sunamura, M. Mizuno, H. Kawaura, T. Hasegawa, K. Terabe, T. Nakayama, M. Aono. EEE J. Solid State Circuits 40, 1, 168 (2005).

[6] A.I. Gusev, S.I. Sadovnikov. Mater. Lett. 188, 351 (2017). 
[7] А.И. Гусев, С.И. Садовников, А.В. Чукин, А.А. Ремпель. ФТT 58, 2, 246 (2016).

[8] S.I. Sadovnikov, A.I. Gusev, A.V. Chukin, A.A. Rempel. Phys. Chem. Chem. Phys. 18, 6, 4617 (2016).

[9] С.И. Садовников, А.И. Гусев. ФТТ 59, 9, 1863 (2017).

[10] S.I. Sadovnikov, A.I. Gusev. J. Therm. Anal. Calorimetry 130, 2, 1155 (2018).

[11] A.I. Gusev, S.I. Sadovnikov. Thermochim. Acta 660, 1 (2018).

[12] Landolt-Börnstein. Group III Condensed Matter. Numerical Data and Functional Relationships in Science and Technology. Volume 41C. Semiconductors. Non-Tetrahedrally Bonded Elements and Binary Compounds I. Silver sulfide $\left(\mathrm{Ag}_{2} \mathrm{~S}\right)$ lattice and further properties, alpha-modification / Eds O. Madelung, U. Rössler, M. Schulz. Springer, Berlin (1998).

[13] https://materialsproject.org/materials/mp-610517/

[14] S.I. Sadovnikov, A.I. Gusev, A.A. Rempel. Superlat. Microstr. 83, 35 (2015).

[15] S.I. Sadovnikov, A.I. Gusev, A.A. Rempel. Phys. Chem. Chem. Phys. 17, 19, 12466 (2015).

[16] S.I. Sadovnikov, A.I. Gusev. Europ. J. Inorg. Chem. 2016, 31, 4944 (2016).

[17] N.W. Ashcroft, N.D. Mermin. Solid State Physics. Cornell University, N.Y.-Chicago-London (1976). 826 p.

[18] T. Blanton, S. Misture, N. Dontula, S.Zdzieszynski. Powd. Diffraction 26, 2, 110 (2011).

[19] G.D. Holah, J.S. Webb, H. Montgomery. J. Phys. 7, 21, 3875 (1974).

[20] D.J. Lockwood, H. Montgomery. J. Phys. C. 8, 19, 3241 (1975).

[21] M.H. Grimsditch, G.D. Holah, Phys. Rev. B 12, 10, 4377 (1975).

[22] C. Carlone, D. Olego, A. Jayaraman, M. Cardona. Phys. Rev. B 22, 8, 3877 (1980).

[23] Landolt-Börnstein. Group III Condensed Matter. Numerical Data and Functional Relationships in Science and Technology. V. 41E. Ternary Compounds, Organic Semiconductors. Silver gallium sulfide $\left(\mathrm{AgGaS}_{2}\right)$ phonon wavenumbers, Grüneisen parameter, piezoelectric constant / Eds O. Madelung, U. Rössler, M. Schulz. Springer, Berlin (2000).

[24] P. Kistaiah, C.V. Reddy, V.P. Kumar, P.V. Reddy, S. Venkanna. J. Alloys Comp. 397, 1-2, 192 (2005).

[25] В.Д. Фараджев, З.А. Искендерзаде, Е.К. Касумова. Е.М. Курбанов. Неорган. материалы 41, 9, 1042 (2005).

[26] В.Н Беломестных, Е.П Теслева. Изв. Томск. политехн. унта 306, 5, 8 (2003).

[27] Д.С. Сандитов, В.Н Беломестных. ЖТФ 81, 11, 77 (2011).

[28] R. Gaillac, P. Pullumbi, F.-X. Coudert. J. Phys.: Cond. Matter 28, 27, 275201 (2016).

[29] A. Jain, S. Ping Ong, G. Hautier, W. Chen, W.D. Richards, S. Dacek, S. Cholia, D. Gunter, D. Skinner, G. Ceder, K.A. Persson. APL Mater. 1, 1, 011002 (2013).

[30] http://progs.coudert.name/elate/mp?query $=$ mp-610517

[31] S.I. Sadovnikov, A.A. Rempel, A.I. Gusev. Russ. Chem. Rev. 87, 4, 303 (2018).

Редактор Д.В. Жуманов 